\title{
A new point-of-care test for the rapid detection of urinary tract infections
}

\author{
Alyexandra Arienzo ${ }^{1}$. Valentina Cellitti ${ }^{2} \cdot$ Valeria Ferrante $^{2} \cdot$ Francesca Losito $^{2} \cdot$ Ottavia Stalio $^{1} \cdot$ Lorenza Murgia $^{1}$. \\ Rossella Marino ${ }^{3}$. Flavia Cristofano ${ }^{3}$ - Michela Orrù ${ }^{2}$. Paolo Visca ${ }^{1}$. Salvatore Di Somma ${ }^{3} \cdot$ Lorena Silvestri $^{4}$. \\ Vincenzo Ziparo ${ }^{4}$ Giovanni Antonini ${ }^{1,2}$ (I)
}

Received: 4 July 2019 / Accepted: 1 October 2019 / Published online: 9 November 2019

(C) The Author(s) 2019

\begin{abstract}
Urinary tract infections (UTIs) are among the most common infections in all age groups. Fast and accurate diagnosis is essential to ensure a timely and effective therapy. Alongside with reference culture-based methods, several point-of-care tests (POCTs) for early detection of UTIs have been developed, but they have not been significantly implemented in current clinical practice. The Micro Biological Survey (MBS) POCT is a simple test developed by MBS Diagnostics Ltd. (London, UK) for the detection and management of UTIs. The present study has been undertaken to investigate the potentials and limits of the MBS POCT. A total of 349 patients were enrolled in two open-label, monocentric, non-interventional clinical trials in collaboration with an Emergency Medicine department and the outpatient clinic of two hospitals in Rome. Results of urine analysis using the MBS POCT were compared with those of the routine culture-based tests for UTI diagnosis performed by the hospital laboratory. The MBS POCT provided fast results revealing high bacterial count UTIs ( $\geq 10^{5} \mathrm{CFU} / \mathrm{ml}$ ) with $97 \%$ accuracy, $92 \%$ sensitivity, $100 \%$ specificity, 99\% PPV, and 96\% NPV within a 5-h analytical time threshold.
\end{abstract}

Keywords Diagnosis $\cdot$ Infection $\cdot$ POCT $\cdot$ UTI

\section{Introduction}

Urinary tract infections (UTIs) remain a major public health problem being among the most common infections in all age groups. The global burden of UTIs is rising, with $16.1 \%$ increase in age-standardized incidence between 1990 and 2013 $[1,2]$. UTIs are also the most common type of healthcareassociated infection, among which $75 \%$ are associated with

Electronic supplementary material The online version of this article (https://doi.org/10.1007/s10096-019-03728-3) contains supplementary material, which is available to authorized users.

Giovanni Antonini

giovanni.antonini@uniroma3.it

1 Science Department, Università degli Studi Roma Tre, Rome, Italy

2 Interuniversity Consortium "Istituto Nazionale Biostrutture e Biosistemi” (INBB), Rome, Italy

3 Emergency Medicine, Department of Medical-Surgery Sciences and Translational Medicine, Università La Sapienza of Rome, Azienda Ospedaliera Sant'Andrea, Rome, Italy

4 Istituto Dermopatico dell'Immacolata, Rome, Italy a urinary catheter, and approximately $20 \%$ are cause of bacteremic complications $[3,4]$.

The current standard for UTI diagnosis is urine culture, followed by antibiotic susceptibility testing (AST) of a midstream, clean-catch urine specimen. UTI patients are empirically treated with antibiotics, and guidelines recommend starting antibiotic treatment before urine culture and AST results become available, delaying by ca. $48 \mathrm{~h}$ the initiation of targeted antibacterial therapy [5-7]. Due to the continually changing rates of antimicrobial resistance, empiric treatments do not ensure appropriate stewardship and can result in therapeutic failure [7-9]. Therefore, the empirical antimicrobial regimen of choice should be based on local resistance patterns, as highlighted in various studies from different countries, to effectively prevent the emergence of multi-drug-resistant uropathogens [10-13]. For these reasons, fast and accurate diagnosis, leading to a rational treatment, is essential to achieve a timely and effective therapy.

A point-of-care test (POCT) is defined as a diagnostic tool applicable near the site of patient care that has the potential to provide an accurate and rapid detection of UTIs. Several POCTs for UTI have been developed and are currently commercially available to detect the presence of bacteria or their 
activity in urine samples, including both culture-based and enzymatic assays, also in automated format [14-18].

The Micro Biological Survey (MBS) POCT is a simple test developed by MBS Diagnostics Ltd. (London, UK) for the management of UTIs $[19,20]$ (Fig. S1 in Supplementary material). It is a culture-based device that allows semiquantitative assessment of viable bacteria concentration. Different from other culture-based methods, it measures the enzymatic activity associated with bacterial metabolism, allowing results to be obtained in short time (ca. $5 \mathrm{~h}$ ).

Performance characteristics of the MBS POCT have preliminarily been investigated in a first prospective diagnostic accuracy evaluation study [21]. A comparative outcome analysis between MBS POCT and reference tests (urine culture and urinalysis, i.e., macroscopic and microscopic examination of urine sediment) was performed, showing that the MBS POCT could detect a suspected UTI within $5 \mathrm{~h}$ with high accuracy (90.2\%), sensitivity (91.2\%), and specificity (89.8\%).

Even considering such promising results, more information concerning potentials and limitations of the method was needed. To this purpose, two trials have been undertaken with the following aims: (i) broaden the preliminary results obtained in the first study and (ii) investigate the cases of discordance between MBS POCT and urine culture reference method, with the aim of pointing out strengths and weaknesses of the MBS POCT. The trials have been conducted between 2015 and 2017 in two different clinical settings in Rome: the Emergency Department of "Azienda Ospedaliera Sant'Andrea" (AOSA) enrolling patients with a severe clinical picture and the outpatient clinic of "Istituto Dermopatico dell'Immacolata" (IDI) enrolling community patients enquiring urine culture for either routine screening (e.g., pregnant women) or clinical suspicion of UTI. These two trials gave the opportunity to broaden the diversity and number of tested patients compared to the first clinical trial [21], highlighting the good performance of the MBS POCT.

\section{Materials and methods}

\section{Study design}

A total of 349 patients were enrolled in two open-label, monocentric, non-interventional clinical trials in collaboration with the Department of Emergency Medicine at AOSA, Rome, and the outpatient clinic at IDI, Rome; 101 and 248 patients respectively were enrolled in the two studies.

Enrollment criteria differed between the two trials, due to the different characteristics of patient attending the two hospitals. Patients admitted at AOSA were enrolled between November 2015 and July 2016. Criteria for enrollment were age $>18$ years, clinical suspicion of UTI including dysuria or acute suprapubic pain and/or costovertebral tenderness or fever and/or cloudy appearance and/or abnormal color of urine, and/or the presence of a catheter left in place for more than $72 \mathrm{~h}$ [21]. Outpatients aged $>18$ enquiring for urine culture were enrolled at IDI from May to December 2017.

Prior to study participation, each patient was asked to read carefully through the patient information sheet and sign the informed consent. Approval of both studies was obtained on 14 Jan 2013 from the Ethical Committee of AOSA and on 25 May 2017 from the Ethical Committee of IDI, constituted according to DM 12 May 2006 following Good Clinical Practice. The authorization was given based on the declaration that the patients were duly informed and consenting. In both trials, the MBS POCT results did not imply any change in the normal diagnostic and therapeutic procedures.

\section{Urine collection}

Midstream urine samples or catheter specimens were collected at AOSA hospital 2-4 h after the last void and kept at $4{ }^{\circ} \mathrm{C}$ for maximum $2 \mathrm{~h}$ prior to analysis. Urine samples at IDI hospital were provided by patients following self-sampling of first morning midstream clean-catch urine specimens [22]. After collection, samples were split into three fractions: one was immediately used for bacterial load assessment with the MBS POCT; one was cultured by the hospital laboratory within half an hour; the last one was split into $1 \mathrm{ml}$ aliquots, each transferred into a 2-ml sterile tube and frozen at $-80^{\circ} \mathrm{C}$ after supplementation with $15 \%$ (vol/vol) glycerol until eventually used for verification analysis.

\section{Hospital laboratory tests}

Urine culture was the reference method used in both hospitals. Urine cultures were performed by the local microbiology laboratory according to Good Laboratory Practice's guidelines: $0.010 \mathrm{ml}$ of undiluted and 100 -fold diluted urine samples were streaked onto blood agar and $\mathrm{BD}^{\mathrm{TM}}$ CHROMagar ${ }^{\mathrm{TM}}$ Orientation Medium (Becton Dickinson GmbH, Heidelberg, DE) plates using a calibrated loop. Colony counts were performed after at least 24 -h incubation at $37{ }^{\circ} \mathrm{C}$. After colony counting, positive results were defined by the presence of $\geq$ $10^{5}$ colony-forming units $(\mathrm{CFU}) / \mathrm{ml}$.

Bacterial identification and antibiogram were performed using the VITEK ${ }^{\circledR}$ MS and VITEK ${ }^{\circledR} 2$ systems (BioMérieux Italia S.p.a., Florence, Italy) with 64-well cartridges for antibiotic susceptibility testing (AST) according to the CLSI recommendations [23].

\section{Bacterial load assessment in urine samples using the MBS POCT}

The MBS POCT device is a colorimetric test designed to be used at the patient's bedside. The test is computer-managed 
and can be battery powered. It provides specific disposable vials for the detection and quantification of bacteria, which contain a non-selective growth medium called Urine Bacterial Quantification (UBQ) required for analysis. The MBS UBQ vials were produced in compliance with requirements set forth in the EU In Vitro Diagnostic Directive. Three independent production batches of UBQ vials were used throughout the first trial, while two more were used in the second trial.

According to the MBS method, each 1-ml urine sample is manually transferred in a UBQ vial using a disposable, graduated, sterile plastic pipette immediately after urine collection. Bacteriuria is automatically detected upon blue to yellow color change of the medium in the reaction vial during time. Criteria for definition of positive and negative results followed results of previous in vitro studies on artificially contaminated urine samples [19], later confirmed by results from the first clinical trial [21], meaning color change within $5.24 \mathrm{~h}$ indicated positivity while slower color change or no color change within analytical timeframe $(24 \mathrm{~h})$ indicated negativity. Vials were incubated in the MBS Multireader, which automatically detects the time for color change, at $37{ }^{\circ} \mathrm{C}$. Analyses were performed in duplicate.

\section{Ex-post verification for bacterial load assessment in urine samples using the MBS POCT}

In case of discordance between the urine culture (reference method) results obtained by the hospital laboratory and the MBS POCT results, a verification analysis was performed by personnel of the Microbiology Laboratory of the Science Department, Roma Tre University, Rome, within 2 days from sampling to investigate the source of discordance. Analyses were repeated with both the reference method and the MBS method using urine samples aliquots that had been stored at $80^{\circ} \mathrm{C}$. Freezing of urine samples did not affect significantly their bacterial load, since no significant decrease of bacterial concentration was observed for frozen samples through 7 days. This trend was observed coherently both using the reference method and the MBS method (data not shown).

Verification analysis of results was performed with the reference method plating $0.010 \mathrm{ml}$ of urine onto non-selective media, including blood agar, CHROM agar, and trypticase soy agar. Colony counting was performed after at least 24-h incubation at $37^{\circ} \mathrm{C}$. Positive results were defined by the presence of $\geq 10^{5} \mathrm{CFU} / \mathrm{ml}$. Positive samples were processed for bacterial identification according to morphology, staining, and biochemical properties of the isolates. Verification analysis of results was performed with the MBS method according to the protocol described above.

In case of concordance between the results obtained with both methods, no further analyses were performed and data was used to carry out statistical analysis. In case of discordance, two possible scenarios were considered (i) when the MBS POCT confirmed a positive result and verification culture showed the absence of a significant bacterial load, the MBS POCT results were definitively considered false positive, and (ii) when the MBS POCT confirmed a negative result and culture showed the presence of a significant bacterial load, results were definitively considered false negative.

\section{Detection of RAA in urine samples}

The residual antimicrobial activity (RAA) test was performed for all discordant samples to check for the presence of antibiotics in urine samples. The detection of residual antibacterial activity was performed using the Bacillus subtilis agar discdiffusion test [24]. An overnight culture of Bacillus subtilis ATCC 6051 was plated on a Mueller-Hinton agar dish. A 13$\mathrm{mm}$ sterile filter paper disk was soaked into the urine sample using sterile forceps and, after the excess liquid was eliminated, the disk was poured on the seeded dish. A positive control (disk containing $10 \mu \mathrm{g}$ ampicillin) and a negative control (blank disk soaked with sterile saline) were also placed on the agar surface. The plates were incubated at $37{ }^{\circ} \mathrm{C}$ for $24 \mathrm{~h}$. The presence of a inhibition halo surrounding the disk soaked in urine similar to that observed around the control antibiotic disk was suggestive of the presence of antibiotic activity in urine.

\section{Diagnostic accuracy evaluation of the MBS POCT}

After the verification step, the final results of the MBS POCT were compared with results of the reference urine culture test. Performance characteristics were evaluated by the receiver operating characteristic (ROC) analysis, using the statistical software MedCalc (Windows version 15.0, MedCalc software, Ostend, Belgium) [25]. This analysis is used to determine the validity of a diagnostic test and to define the optimal cut off limit. The area under the ROC curve (AUC) is a measure of how well a parameter can distinguish between two diagnostic groups, i.e., in this investigation, patients with and without a UTI. An area under the curve equal to 1 is that of a test displaying $100 \%$ sensitivity and $100 \%$ specificity [26, 27].

\section{Results}

\section{Patient characteristics}

The 101 patients attending AOSA Emergency Medicine Department had the following characteristics: $56(55 \%)$ women, 45 (45\%) men; mean age 78 years (range 44-96); 79 (78\%) catheterized; $77(76 \%)$ were admitted with an ongoing antibiotic therapy and the most frequently used antibiotics 
were piperacillin-tazobactam (34\%), ciprofloxacin (26\%) levofloxacin (21\%), and cefotaxime (14\%), seldom used in combination with other drugs. The 248 outpatients attending the IDI displayed the following characteristics: 173 (70\%) women, 75 (30\%) men; mean age 65 years (range 18-93); six $(2 \%)$ catheterized. Of the patients enrolled for the study, only three $(1 \%)$ were admitted with an ongoing antibiotic therapy.

\section{Urine culture results}

Urine culture routinely performed by hospital laboratories yielded 124 positive results and 220 negative results. Urine culture was not available in three cases, and thus, data from those patients were not included in the statistical analysis. Polymicrobial infections accounted for 21 cases $(8 \%)$. Bacterial isolates were Escherichia coli $(n=20)$, Enterococcus faecalis $(n=16)$, Pseudomonas aeruginosa $(n=7)$, Klebsiella pneumoniae $(n=5)$, Proteus mirabilis $(n=5)$, Enterococcus faecium $(n=2)$, Klebsiella oxytoca $(n=2)$, Achromobacter xylosoxidans $(n=1)$, Enterobacter cloacae $(n=1)$, Morganella morganii $(n=1)$, Staphylococcus aureus $(n=1)$, and Citrobacter freundii $(n=1)$. The most common polymicrobic associations involved E. coli and E. faecalis $(n=7)$.

Culture-positive results were obtained from 17 out of 77 urine samples from patients undergoing antibiotic therapy (22\%), and from 107 out of 267 samples from patients untreated with antibiotics $(40 \%)$.

\section{MBS POCT results}

A positive result (median $2.0275,95 \%$ CI 1.7800 to 2.2388 ) was obtained for 114 out of 344 samples analyzed (33\%). The average time for color change of positive samples was $2.03 \mathrm{~h}$ (range $0.66-5.24 \mathrm{~h}$ ). No color change was observed for 85 urine samples, while for 145 samples, a color change was observed between 5.25 and $26 \mathrm{~h}$, and thus reported as negative. MBS POCT-positive results were obtained from 13 out of 77 urine samples from patients undergoing antibiotic therapy (17\%), and from 101 out of 267 urine samples from patients untreated with antibiotics (38\%). Two urine samples displayed evident macro hematuria which interfered with MBS Multireader measurements. Therefore, the MBS POCT was considered not applicable and the two samples were discarded.

\section{Diagnostic accuracy of the MBS POCT}

Discordance between the results obtained with the reference culture method and the MBS POCT was observed for 19 urine samples out of 344 analyzed (6\%). Ex-post verification was therefore carried out on frozen urine samples. Definitive results for all samples following the verification analyses, with the exclusion of two samples with macrohematuria and three samples lacking urine culture results, are summarized in Table 1. Sensitivity, specificity, and positive and negative predictive values of the MBS POCT were $92 \%, 100 \%, 99 \%$, and $96 \%$, respectively.

To assess the diagnostic accuracy and optimize the incubation time of the MBS POCT, ROC analysis was performed considering the final results obtained after verification analyses. A total of 344 samples were included in the analysis. Quantitative results, in terms of time taken for the vials to change color, were compared to those of urine culture (either positive or negative). Upon ROC curve analysis, the AUC of the MBS POCT was 0.987 (95\% CI 0.973 to 1.000) (Fig. 1), and the associated criterion was $5.24 \mathrm{~h}$. In addition, it should be underlined that almost $50 \%$ of culture-confirmed infections were detected within $2 \mathrm{~h}$ by the MBS POCT (Fig. 2).

Further analysis of discordant cases highlighted an intermediate time zone, ranging from 5.25 to $7 \mathrm{~h}$, which included most of the discordant results. Such intermediate zone is critical, as it comprises samples with borderline bacterial load (around $10^{5} \mathrm{CFU} / \mathrm{ml}$ ). Therefore, further concordance analysis was performed by combining MBS POCT results for color change times $\leq 5.24 \mathrm{~h}$ and $\geq 7 \mathrm{~h}$ (Table 2). Sensitivity and specificity considering the combined dataset were $98 \%$ and $100 \%$, respectively, whereas positive and negative predictive values reached $100 \%$ and $99 \%$ respectively. The AUC obtained from the ROC analysis performed on this dataset was 0.992 (95\% CI 0.979 to 1.000 ), and the associated criterion was again 5.24 h (Fig. 3). Categorization of MBS POCT results for cut-off values of 5.24 and $7 \mathrm{~h}$ are shown in Fig. 4.

\section{Discussion}

In this work, the diagnostic accuracy of the MBS POCT was investigated in different clinical settings in order to highlight the potential benefits and limits of the new method in comparison with conventional urine culture, considered as the gold standard. Different patient cohorts were investigated, i.e., a population of elderly patients admitted to the hospital with

Table 1 Summary of definitive results obtained upon verification by urine culture and MBS POCT analysis (dataset from 344 samples)

\begin{tabular}{lllll}
\hline & \multicolumn{4}{l}{ Urine culture (cut-off $\left.10^{5} \mathrm{CFU} / \mathrm{ml}\right)$} \\
\cline { 2 - 5 } & & Positive & Negative & Total \\
\hline \multirow{2}{*}{ MBS POCT (cut-off 5.24 h) } & Positive & 113 & 1 & 114 \\
& Negative & 10 & 220 & 230 \\
& Total & 123 & 221 & $344^{\mathrm{a}}$ \\
\hline
\end{tabular}

${ }^{\mathrm{a}}$ Two samples with macro hematuria and three lacking urine culture were excluded from the analysis 


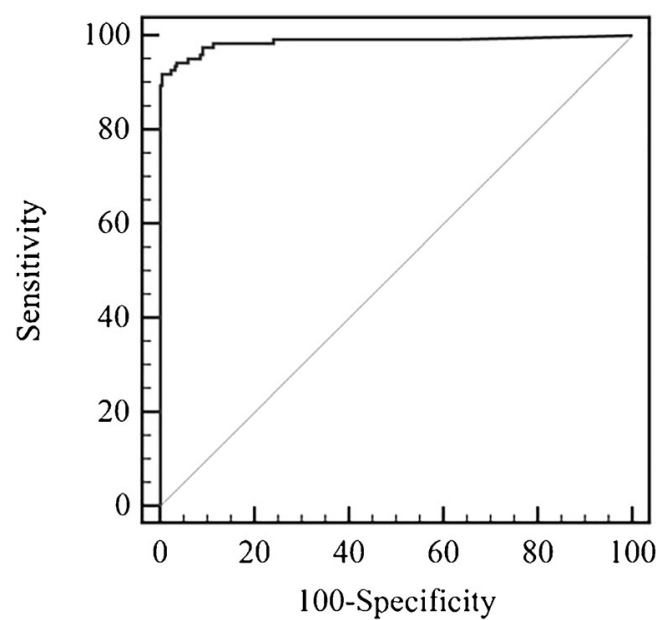

Fig. 1 ROC analysis of MBS POCT results $(n=344)$. The ROC curve shows an $\mathrm{AUC}=0.987$ with $95 \%$ confidence interval from 0.973 to 1.000 (dotted line)

underlying disease, often catheterized or undergoing antibiotic therapy (AOSA hospital), together with a cohort of outpatients enquiring for urine microbiological analysis, hence younger, non-catheterized, and without ongoing antibiotic treatment (IDI hospital). This offered the opportunity to define the MBS POCT performance in different clinical frameworks.

Results reported in this study reveal an overall high accuracy, sensitivity, and specificity of the MBS POCT. ROC assessment of the MBS POCT revealed 97\% accuracy, and the associated criterion was $5.24 \mathrm{~h}$, coherent with a preliminary study [21]. Moreover, faster response (2 h) was observed for nearly $50 \%$ of culture-confirmed UTIs, coherent with the basic principle of the method that implies a reverse correlation between the time of response and the bacterial load in the sample [28, 29]. UTI is the main cause of sepsis in nearly $30 \%$ of all septic patients [30], particularly in the elderly population [31]. This type of patients predominated in the
Table 2 Summary of definitive results obtained upon verification by urine culture and MBS POCT analysis considering samples showing color change $\leq 5.24 \mathrm{~h}$ and $\geq 7 \mathrm{~h}$ (dataset from 303 samples)

\begin{tabular}{lllll}
\hline & \multicolumn{3}{l}{ Urine culture (cut-off $\left.10^{5} \mathrm{CFU} / \mathrm{ml}\right)$} \\
\cline { 2 - 5 } & & Positive & Negative & Total \\
\hline \multirow{2}{*}{ MBS POCT (cut-off 5.24 h) } & Positive & 113 & 0 & 113 \\
& Negative & 2 & 188 & 190 \\
& Total & 115 & 188 & $303^{\text {a }}$ \\
\hline
\end{tabular}

${ }^{\mathrm{a}}$ Two samples with macro hematuria and three lacking urine culture were excluded from the analysis

Emergency Medicine Department of AOSA; this clinical setting would certainly benefit from a diagnostic tool for early UTI diagnosis, possibly flanking the very first intervention in the "golden hour."

By comparison with the "golden standard," MBS POCT true positive and true negative results were mostly comprised within two categories: color change occurring within $5.24 \mathrm{~h}$ and after $7 \mathrm{~h}$, respectively. This was confirmed by ROC analysis, which showed very high sensitivity and specificity values (98\% and 100\% respectively), and $99 \%$ accuracy for the above categories. Urines from 41 patients out of $344(12 \%)$ did not fall within these categories, showing vial color change between 5.25 and $7 \mathrm{~h}$. These patients showed a borderline bacterial load, as inferred from viable counts between $10^{4}$ and $10^{5} \mathrm{CFU} / \mathrm{ml}$ in reference urine culture (Table S1 in Supplementary material); setting clear thresholds in these cases is difficult, so that MBS POCT results are uncertain. Irrespective of the analytical method, UTI diagnosis in such patients must be supported by objective examination, taking into account patient symptoms and clinical picture.
Fig. 2 Effect of detection time on MBS POCT positivity. Histograms show detection frequencies (\%) of MBS POCTpositive results at hourly intervals

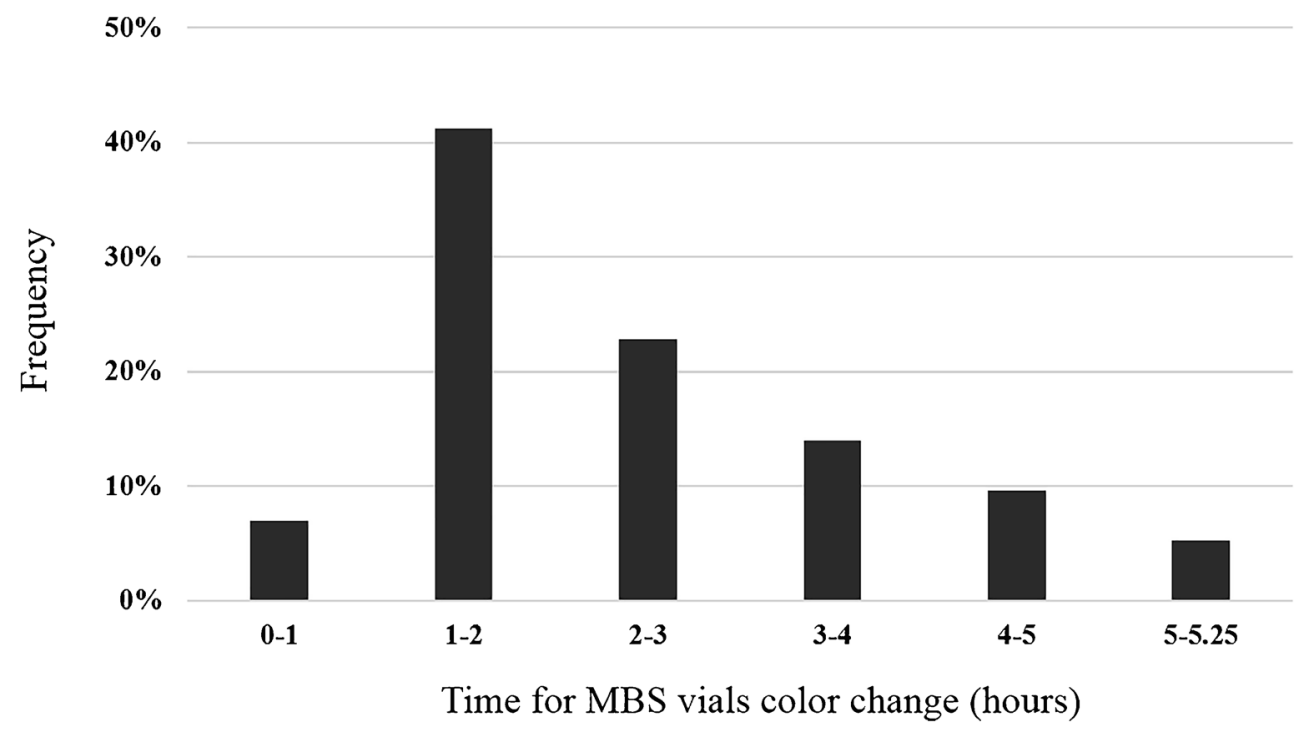




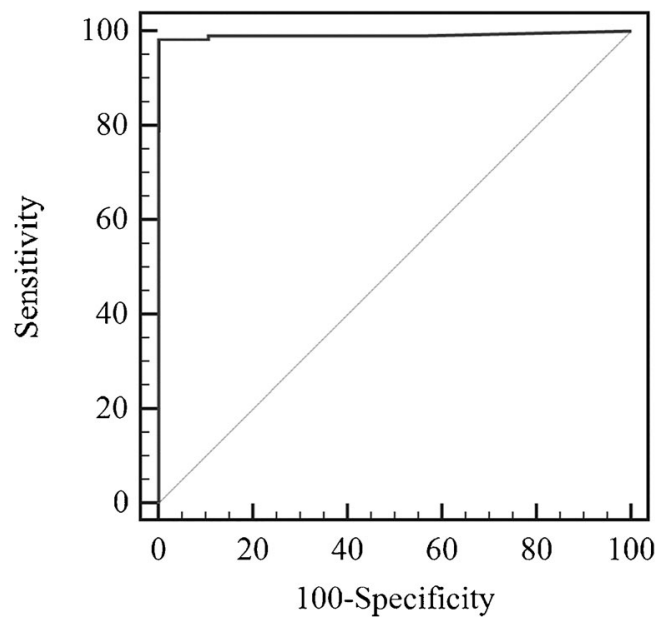

Fig. 3 ROC analysis of MBS POCT results $(n=303)$. The ROC curve shows an AUC $=0.992$ with $95 \%$ confidence interval from 0.979 to 1.000 (dotted line)

MBS POCT results could be of guidance for patient management. For instance, antibiogram and treatment should be secured for patients falling in the $\leq 5.24 \mathrm{~h}$ category, as opposed to patients falling in the $>7 \mathrm{~h}$ category in which UTI could be ruled out. Of note, monitoring of vials which do not change color within $5.24 \mathrm{~h}$ should not be discontinued until $7 \mathrm{~h}$, in order to detect slow-growing bacteria and virtually low bacterial load $\left(<10^{5} \mathrm{CFU} / \mathrm{ml}\right)$, as this could be significant for some categories of patients. Indeed, the bacterial concentration threshold should be set taking into account patient's age, sex, and clinical picture [32]. As important as positive predictive value, MBS POCT showed a high negative predictive value (96\%), being able to rule out culture-negative patients. As a whole, results obtained in this study have

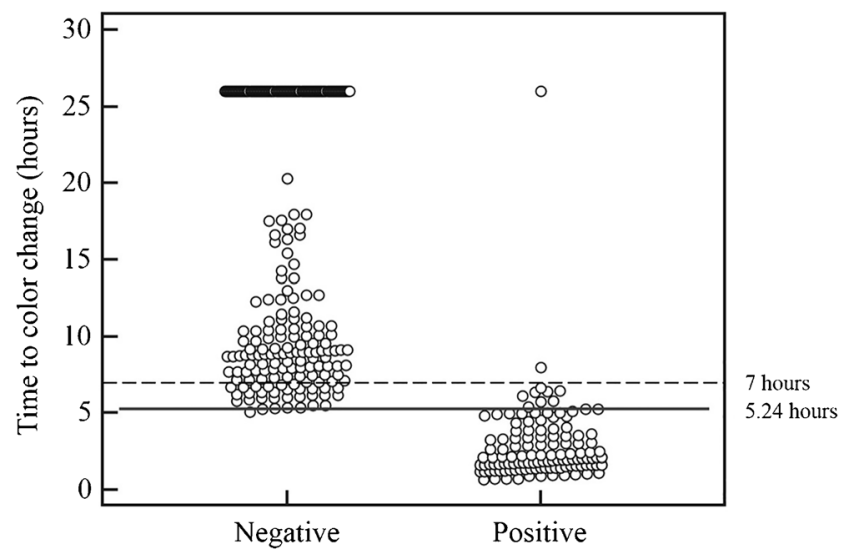

Fig. 4 Dot plot analysis of MBS POCT results $(n=344)$. Distribution of positive and negative results with a threshold limit of $5.24 \mathrm{~h}$ (sensibility $91.9 \%$, specificity $99.5 \%$ ). Lines denote the threshold limits, defining three categories: negative (vial color change $>7 \mathrm{~h}$ ), uncertain (vial color change between 5.25 and $7 \mathrm{~h}$ ), positive (vial color change $\leq 5.24 \mathrm{~h}$ ) highlighted the robustness of the MBS POCT for the detection of suspected UTIs and suggest how its rapidity, simplicity, and user-friendliness could represent key advantages for the clinical management of patients before a UTI is confirmed by culture-based laboratory methods. By comparison with available devices for UTI detection [33-37], it appears that the MBS POCT could place well among other culture-based devices thanks to its ability to combine short analytical time and high accuracy.

This work also highlights few limitations of the MBS POCT, which appear inherent with the analytical principles of the method. First, since bacterial growth is detected through a colorimetric assay, in the presence of a heavy hematuria, vial color change is biased by the color of the sample, impairing signal detection. This condition is, however, rarely observed in uncomplicated UTIs and was found in our trials in only two cases $(0.6 \%)$, due to the particular population enrolled in the AOSA study. Furthermore, since the method measures the metabolic activity of bacteria, ongoing antibiotic therapy, hence the presence of antibiotics in urine, affects the viability and/or the metabolic state of bacteria in the sample. This particular condition has been observed in only one case $(0.3 \%)$. In this case, antibiotic therapy was administered shortly before urine sampling and the infecting bacteria were susceptible. It should be taken into account that urine samples are 10-fold diluted in the MBS POCT, and this can cause significant antibiotic carryover, as opposed to agar plate counting. This situation could have some intriguing clinical implications: when information on ongoing antibiotic therapy is available for a patient, a negative MBS POCT result is suggestive of no infection in urine or successful therapy, while a positive result should warn the clinician about the possible failure of ongoing therapy.

On a different note, setting thresholds for significant UTI is challenging, and the clinical value of a unique $10^{5} \mathrm{CFU} / \mathrm{ml}$ cutoff concentration has been questioned. A major concern is the underestimation of infections that could arise from this approach, since low urinary bacterial counts may hold clinical significance in particularly vulnerable categories of patients [38]. Low bacterial counts $\left(10^{2} \mathrm{CFU} / \mathrm{ml}\right)$ can be significant depending on the bacterial agent and patient clinical picture [39], but these infections are usually untreated, though they can degenerate into high-count UTIs in a few days [40]. On this basis, the MBS POCT has some limitations, in that the detection of low-count bacteriuria $\left(<10^{4} \mathrm{CFU} / \mathrm{ml}\right)$ is not compatible with the rapidity of the test (i.e., detection time $<7 \mathrm{~h}$ ). Indeed, low bacterial counts can be detected with the MBS POCT device within $15 \mathrm{~h}$ (Fig. S2 in Supplementary material), though this does not represent an advantage over routine culture. Another limitation of the MBS POCT is the inability to identify the pathogen(s) involved in the infection, and this could lead to antibiotic misuse. Nevertheless, the MBS POCT 
could be considered as an enrichment culture that could undergo further analysis according to resource availability, e.g., by direct MALDI-TOF analysis for monomicrobic samples or routine plating for polymicrobic associations, as inferred by microscopy examination of Gram stains of MBS POCTpositive samples [41].

In conclusion, the MBS POCT is a simple and efficient diagnostic tool, which holds promise for improving UTI detection at the patient bedside. Notably, it provides a precise negative predictive value in few hours, allowing early exclusion of high bacterial load UTI diagnosis, with a positive impact on patient management, laboratory workload, and healthcare-associated costs.

Acknowledgments The authors gratefully acknowledge Dr. Andreas Bergmann (Sphingotec GmbH, 16761 Hennigsdorf, Germany), Dr. Metod Miklus, Dr. Robert Hellmich, and Dr. Paul Scigalla (Oncoprevent GmbH, 16761 Hennigsdorf, Germany) for the helpful discussions, and MBS Diagnostics Ltd (London, UK) as vial providers.

Author's contributions GA and PV conceived the study. GA and SDS designed the study and coordinated the research. VC, VF, RM, and MO performed the experimental work. AA, VC, and VF collected the data. AA and LM analyzed the data and drafted the manuscript. VC, VF, FL, and OS contributed to interpret the data and revise the article. GA, SDS, and PV critically reviewed the article. The authors warrant that all the authors have contributed substantially to the manuscript and approved the final submission.

Funding information This work has been partially supported by Marjan Miklus Stiftung, Zur Forderung der Forschung, 16761 Hennigsdorf, Germany, by Waltraut Bergmann Stiftung, Zur Forderung von Wissenschaft und Forschung, 16556 Borgsdorf, Germany, and by two national grants: Sviluppo Lazio spa, 00198 Roma, Italy [grant number FILAS- RS-2009-1026], and the National Funding for Centers of Excellence (Science Department, Roma Tre University - MIUR, Articolo 1, Commi 314-337 Legge 232/2016).

\section{Compliance with ethical standards}

Conflict of interest The authors declare that they have no conflict of interest.

Informed consent Informed consent was obtained from all individual participants included in the study.

Ethical approval All procedures performed in studies involving human participants were in accordance with the ethical standards of the institutional and/or national research committee (from Ethical Committee of Azienda Ospedaliera Sant'Andrea on 2019/01/14; from Ethical Committee of Istituto Dermopatico dell'Immacolata on 2017/05/25) and with the 1964 Helsinki declaration and its later amendments or comparable ethical standards.

Open Access This article is distributed under the terms of the Creative Commons Attribution 4.0 International License (http:// creativecommons.org/licenses/by/4.0/), which permits unrestricted use, distribution, and reproduction in any medium, provided you give appropriate credit to the original author(s) and the source, provide a link to the Creative Commons license, and indicate if changes were made.

\section{References}

1. Hanna-Wakim RH, Ghanem ST, El Helou MW, Khafaja SA, Shaker RA, Hassan SA, Saad RK, Hedari CP, Khinkarly RW, Hajar FM, Bakhash M, El Karah D, Akel IS, Rajab MA, Khoury M, Dbaibo GS (2015) Epidemiology and characteristics of urinary tract infections in children and adolescents. Front Cell Infect Microbiol 5:45. https://doi.org/10.3389/fcimb.2015.00045

2. Flores-Mireles AL, Walker JN, Caparon M, Hultgren SJ (2015) Urinary tract infections: epidemiology, mechanisms of infection and treatment options. Nat Rev Microbiol 13(5):269-284. https:// doi.org/10.1038/nrmicro3432

3. Lai KK, Fontecchio SA (2002) Use of silver-hydrogel urinary catheters on the incidence of catheter-associated urinary tract infections in hospitalized patients. Am J Infect Control 30(4):221-225

4. Gould CV, Umscheid CA, Agarwal RK, Kuntz G, Pegues DA (2010) Guideline for prevention of catheter-associated urinary tract infections. Infect Control Hosp Epidemiol 31(4):319-326. https:// doi.org/10.1086/651091

5. Thuan PL, Loren GM (2001) Empirical therapy for uncomplicated urinary tract infections in an era of increasing antimicrobial resistance: a decision and cost analysis. Clin Infect Dis 33(5):615-621. https://doi.org/10.1086/322603

6. Wilson MI, Gaido L (2004) Laboratory diagnosis of urinary tract infections in adult patients. Clin Infect Dis 38:1150

7. Foxman B (2010) The epidemiology of urinary tract infection. Nat Rev Urol 7:653-660

8. Maki DG, Tambyah PA (2001) Engineering out the risk for infection with urinary catheters. Emerg Infect Dis 7(2):342-347

9. Arason VA, Sigurdsson JA (2010) The problems of antibiotic overuse. Scand J Prim Health Care 28(2):65-66. https://doi.org/10. 3109/02813432.2010.487652

10. Linhares I, Raposo T, Rodrigues A, Almeida A (2015) Incidence and diversity of antimicrobial multidrug resistance profiles of uropathogenic bacteria. BioMed Research International 2015, Article ID 354084:11 pages. https://doi.org/10.1155/2015/354084

11. Bryce A, Hay AD, Isabel Lane IF, Thornton HV, Wootton M, Costelloe C (2016) Global prevalence of antibiotic resistance in paediatric urinary tract infections caused by Escherichia coli and association with routine use of antibiotics in primary care: systematic review and meta-analysis. BMJ 352:1939. https://doi.org/10. 1136/bmj.i939

12. Gupta K, Sahm DF, Mayfield D, Stamm WE (2001) Antimicrobial resistance among uropathogens that cause community-acquired urinary tract infections in women: a nationwide analysis. Clin Infect Dis 33(1):89-94. https://doi.org/10.1086/320880

13. Mandal J, Acharya NS, Buddhapriya D, Parija SC (2012) Antibiotic resistance pattern among common bacterial uropathogens with a special reference to ciprofloxacin resistant Escherichia coli. Indian J Med Res 136(5):842-849

14. Baerheim A (2012) Empirical treatment of uncomplicated cystitis. Scand J Prim Health Care 30(1):1-2. https://doi.org/10.3109/ 02813432.2012.649629

15. NIHR Diagnostic Evidence Cooperative Oxford (2016) Point-ofcare testing for urinary tract infections. Horizon Scan Report 0045

16. Waisman Y, Zerem E, Amir L, Mimouni M (1999) The validity of the uriscreen test for early detection of urinary tract infection in children. Pediatrics 104(4)

17. Holm A, Cordoba G, Sørensen TM, Jessen LR, Siersma V, Bjerrum L (2015) Point of care susceptibility testing in primary care - does it lead to a more appropriate prescription of antibiotics in patients with uncomplicated urinary tract infections? Protocol for a randomized controlled trial. BMC Fam Pract 16:106. https://doi.org/10.1186/ s12875-015-0322-x 
18. Schot MJC, Van Delft S, Kooijman-Buiting AMJ, de Wit NJ, Hopstaken RM (2015) Analytical performance, agreement and user-friendliness of six point-of-care testing urine analyzers for urinary tract infection in general practice. BMJ Open 5(5):e006857. https://doi.org/10.1136/bmjopen-2014-006857

19. Bottini G, Losito F, Arienzo A, Priolisi FR, Mari A, Visca P, Antonini G (2013) A new method for microbiological analysis that could be used for point-of-care testing (POCT). TOEMJ 5:13-15

20. Arienzo A, Losito F, Bottini G, Priolisi FR, Mari A, Visca P, Antonini G (2014) A new device for the prompt diagnosis of urinary tract infections. Clin Chem Lab Med 52(10):1507

21. Arienzo A, Cellitti V, Ferrante V, Losito F, Stalio O, Cristofano F, Marino R, Magrini L, Santino I, Mari A, Visca P, Di Somma S, Antonini G (2016) A pilot clinical trial on a new point-of-care test for the diagnosis and fast management of urinary tract infections in the emergency department. Int J Clin Med Microbiol 1:107

22. Clinical and Laboratory Standards Institute (2001) Urinalysis and collection, transportation, and preservation of urine specimens; Approved Guideline - Second Edition. Vol. 21. No. 19. Document GP-16A2

23. Wayne, PA (2013) Clinical and laboratory standards institute. Performance standards for antimicrobial susceptibility testing; M100-MS23

24. Hoppe JE, Schlegel M (1999) Detection of antibacterial activity in the urine of children: a comparison of three tests. Klin Padiatr 211(2):79-82

25. DeLong ER, DeLong DM, Clarke-Pearson DL (1988) Comparing the areas under two or more correlated receiver operating characteristic curves: a nonparametric approach. Biom J 44:837-845

26. Griner PF, Mayewski RJ, Mushlin AI, Greenland P (1981) Selection and interpretation of diagnostic tests and procedures. Principles and applications. Ann Intern Med 94(4 Pt 2):557-592

27. Zweig MH, Campbell G (1993) Receiver-operating characteristic (ROC) plots: a fundamental evaluation tool in clinical medicine. Clin Chem 39(4):561-577

28. Bottini G, Losito F, De Ascentis A, Priolisi FR, Mari A, Antonini G (2011) Validation of the micro biological survey method for total viable count and $E$. coli in food samples. Am J Food Technol 6(11): 951-962. https://doi.org/10.3923/ajft.2011.951.962

29. Losito F, Bottini G, De Ascentis A, Priolisi FR, Mari A, Tarsitani G, Antonini G (2012) Qualitative and quantitative validation of the micro biological survey method for Listeria spp., Salmonella spp., Enterobacteriaceae and Staphylococcus aureus in food samples. Am J Food Technol 7(6):340-351. https://doi.org/10.3923/ajft. 2012.340.351

30. Wagenlehner FME, Pilatz A, Weidner W, Naber KG (2015) Urosepsis: overview of the diagnostic and treatment challenges.
Microbiol Spectr 3(5). https://doi.org/10.1128/microbiolspec.UTI0003-2012

31. Holloway WJ (1986) Management of sepsis in the elderly. Am J Med 80(6B):143-148

32. Grabe M, Bartoletti R, Bjerklund Johansen TE, Cai T, Çek M, Köves B, Naber KG, Pickard RS, Tenke P, Wagenlehner F, Wullt B (2015) Guidelines on urological infections. European Association of Urology

33. Bongard E, Frimodt-Møller N, Gal M et al (2015) Analytic laboratory performance of a point of care urine culture kit for diagnosis and antibiotic susceptibility testing. Eur J Clin Microbiol Infect Dis 34:2111. https://doi.org/10.1007/s10096-015-2460-4

34. Holm A, Cordoba G, Sørensen TM, Jessen LR, Frimodt-Møller N, Siersma V, Bjerrum L (2017) Clinical accuracy of point-of-care urine culture in general practice. Scand J Prim Health Care 35(2): 170-177. https://doi.org/10.1080/02813432.2017.1333304

35. Butler CC, Francis NA, Thomas-Jones E, Longo M, Wootton M, Llor C, Little P, Moore M, Bates J, Pickles T, Kirby N, Gillespie D, Rumsby K, Brugman C, Gal M, Hood K, Verheij T (2018) Point-ofcare urine culture for managing urinary tract infection in primary care: a randomised controlled trial of clinical and cost-effectiveness. Br J Gen Pract 68(669):e268-e278. https://doi.org/10.3399/ bjgp18X695285

36. Yagupsky P, Rider M, Peled N (2000) Clinical evaluation of a novel chromogenic agar dipslide for diagnosis of urinary tract infections. Eur J Clin Microbiol Infect Dis 19(9):694-698

37. Rosenberg $M$ et al (1992) Initial testing of a novel urine culture device. J Clin Microbiol 30(10):2686-2691

38. Stark RP, Maki DG (1984) Bacteriuria in the catheterized patient. What quantitative level of bacteriuria is relevant? N Engl J Med 311(9):560-564

39. Brecher SM (2016) Complicated urinary tract infections: what's a lab to do? J Clin Microbiol 54(5):1189-1190. https://doi.org/10. 1128/JCM.00370-16

40. Arav-Boger R, Leibovici L, Danon YL (1994) Urinary tract infections with low and high colony counts in young women. Spontaneous remission and single-dose vs multiple-day treatment. Arch Intern Med 154(3):300-304

41. Pinault L, Chabrière E, Raoult D, Fenollar F (2019) Direct identification of pathogens in urine by use of a specific matrix-assisted laser desorption ionization-time of flight spectrum database. J Clin Microbiol 57(4). https://doi.org/10.1128/JCM.01678-18

Publisher's note Springer Nature remains neutral with regard to jurisdictional claims in published maps and institutional affiliations. 